\title{
VISION BASED HUMAN COMPUTER INTERFACE USING COLOUR DETECTION
}

\author{
Hamza Jasnak', Masoom Chouhan ${ }^{2}$, Shadab Barmare ${ }^{3}$, Zafar Khan ${ }^{4}$ \\ 1, 2, 3,4 Department of Electronics \& Communication Engineering,M.H, Saboo Siddik College of Engineering \\ Maharashtra, India
}

\begin{abstract}
In this paper we have tried to present an approach to Human Computer Interaction (HCI). Here we have tried to control actions associated with mouse. Each mouse actions are associated to a colour pointer. These colour pointer are acquired as input using web camera. The acquired colour pointer are processed using colour detection technique.
\end{abstract}

Keywords: Human Computer Interaction, Web Camera, Colour Detection, Colour Subtraction.

\section{INTRODUCTION}

Nowadays Human Computer Interaction (HCI) is being developed greatly to improve the interaction with the computer. The personal computers (PC's) are well developed which are provided with peripherals like track pads, touch screen to interact with the personal computers (PC's). But these technologies are not cheap, so to make Human Computer Interaction (HCI) cost effective we have to use some peripheral that can also be used for other than Human Computer Interaction (HCI). So as an alternative approach we can use web camera to interact with the personal computers.

\section{RELATED WORKS}

There are many approaches for Human Computer Interaction (HCI) that use web camera. Some of the approach is as follows:

\subsection{HCI Without Using Interface}

In this approach no material is required to interact with the computer

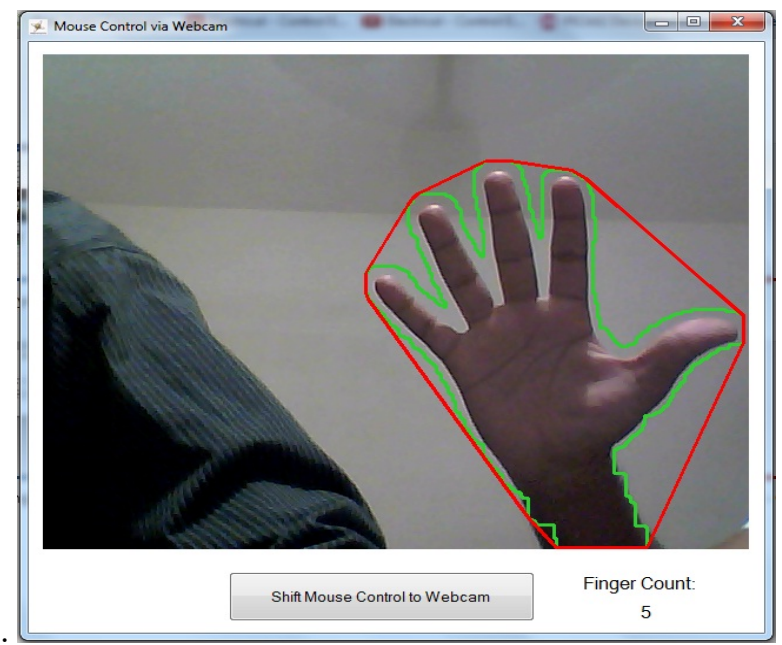

Figure 1. HCI without interface

\subsubsection{Hand Gesture Controlled Mouse}

In this approach no material is required the mouse actions are controlled using plain fingers. Bare hands are used to track the movement of finger in front of the camera. The fingers are detected on basis of skin colour and texture.

\subsection{HCI Using Interface}

In this approach we use some material to interact with the personal computer like markers, data glove, etc.

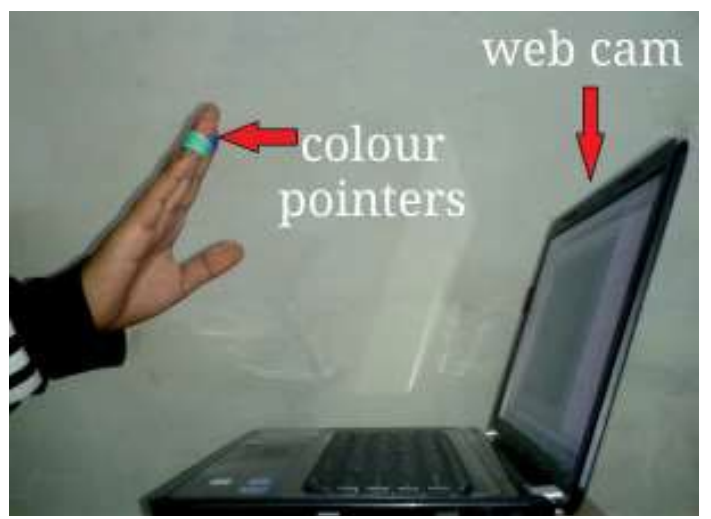

Figure 2. HCI using interface

\subsubsection{Using Colour Markers Or Pointers}

In this approach we use colour pointers to interact with the PC. The colour pointers are placed directly on the finger tips or the fingertips of the gloves can be marked using colour pointer. [2][3]

\section{INTRODUTION TO THE SYSTEM}

In our project we have tried to control the actions of mouse using colour detection. We have controlled mouse actions like cursor movement, clicking actions and scrolling actions. We use web camera to capture real time video. The input real time video is processed frame by frame. The user wears the colour pointers to control the actions of mouse. The 
input real time video is converted into frame and processed frame by frame. The colour is detected using image subtraction algorithm. After detecting colour the system performs various operations to control cursor movement, clicking actions and scrolling action. The detailed working is given below. Only web camera is required to perform the control actions and no other hardware is required. [5]

\section{SYSTEM DISCRIPTION}

Steps followed in our approach are:

1. Get real time video from the web camera.

2. The input video is processed frame by frame.

3. Each frame is flipped to avoid mirror effect.

4. Convert each frame of the video is converted into grayscale image

5. Detection of colour from the input video using image subtraction technique.

6. Convert the detected colour image to binary image.

7. Calculating the size of the pointer and the centroid of the detected colour.

8. Identify the colour detected from the input video.

9. Identify the number of pointers detected.

10. Perform mouse actions associated with each of the colour pointers and number of pointers.[1]

\section{FLOWCHART}

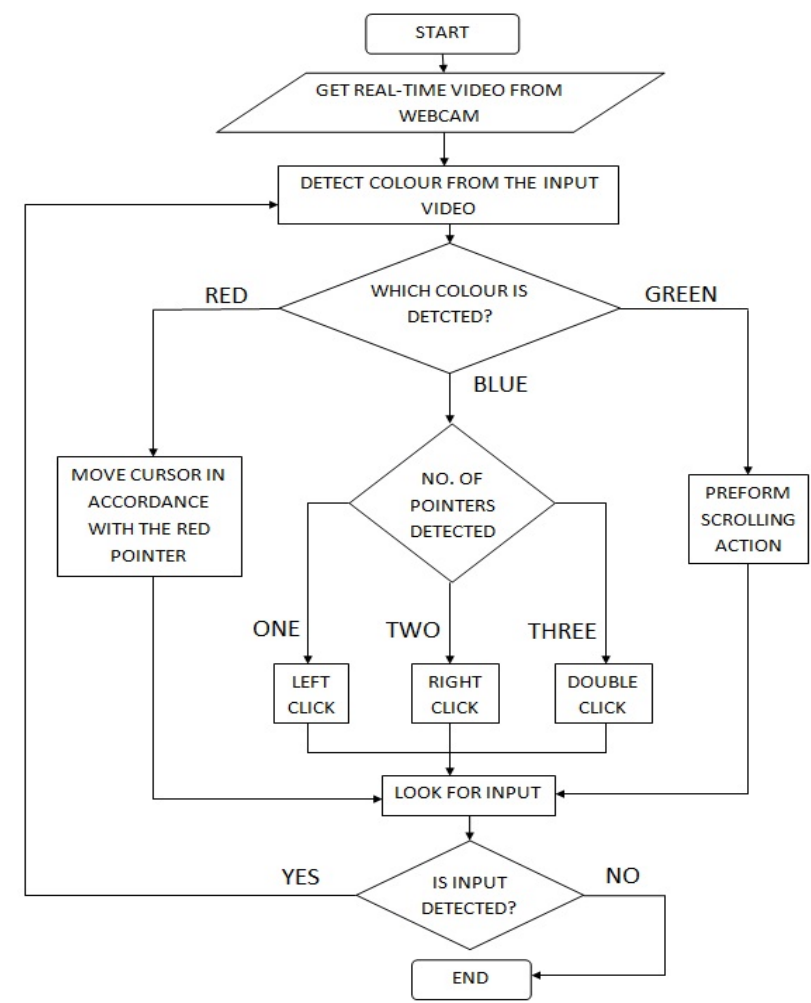

Figure 3. Flowchart

\subsection{Get Real-Time Video From Web Camera}

The computer takes real-time video from web camera as input for HCI. Then the computer starts processing of then video for further actions. After acquiring video it is processed frame by frame. [5]

\subsection{Detection of Colour Pointer}

The camera captures video in an inverted fashion, means that when the pointer moves from left to right the pointer in the video moves from right to left and when the pointer moves from right to left the pointer in the video moves from left to right. The image obtained from the web camera is a mirror image. So in order to move the pointer in video without mirror effect the video is inverted.

The next step is colour extraction and detection. To detect colour from the video is done by colour subtraction technique. The coloured image is combination of three frames red frame, blue frame and green frame. The combinations of these frames are in different ratios for different images. So to detect a particular colour we have to use that particular colour frame from the image. These colour frames are obtained on suppressed channel for red, blue and green. But since these computation is difficult in coloured image so the image is converted to gray-scale image. Then the suppressed channel is subtracted from the gray-scale image. The subtracted image contains the detected colour in the form of a gray patch surrounded by black background.

After extracting the colour from the image, the image containing the gray patch is converted to binary image. When the gray-scale image is converted into binary image, it is stored in the form of a matrix containing values of each pixel. The pixel value range from 0 to 255 , where 0 represents black and 255 represents white. To separate the detected patch distantly we will use thresholding by setting the threshold value to $20 \%$ of the maximum value. Thresholding will make pixel value below threshold level to 0 and pixel value greater than threshold value to 255 so that the detected patch can be distinctly seen.[9][10][11]

\subsection{Tracking of Colour Pointer}

After detecting the colour from the image size of the colour pointer is identified, so that too large objects or too small pointer is not detected. After identifying the contour of the colour pointer a colour blog is put on the detected colour patch. Using the identified contour of the colour pointer the centroid of the colour pointer is calculated. The colour pointer is tracked with the help of the centroid obtained. [12]

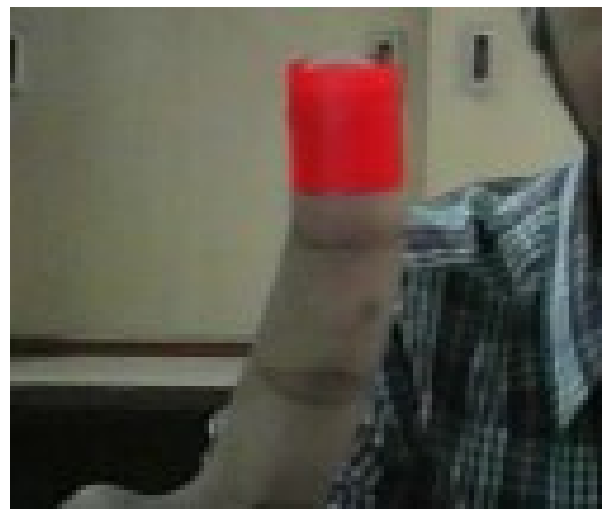

Figure 4. Detection of Red Colour pointer 


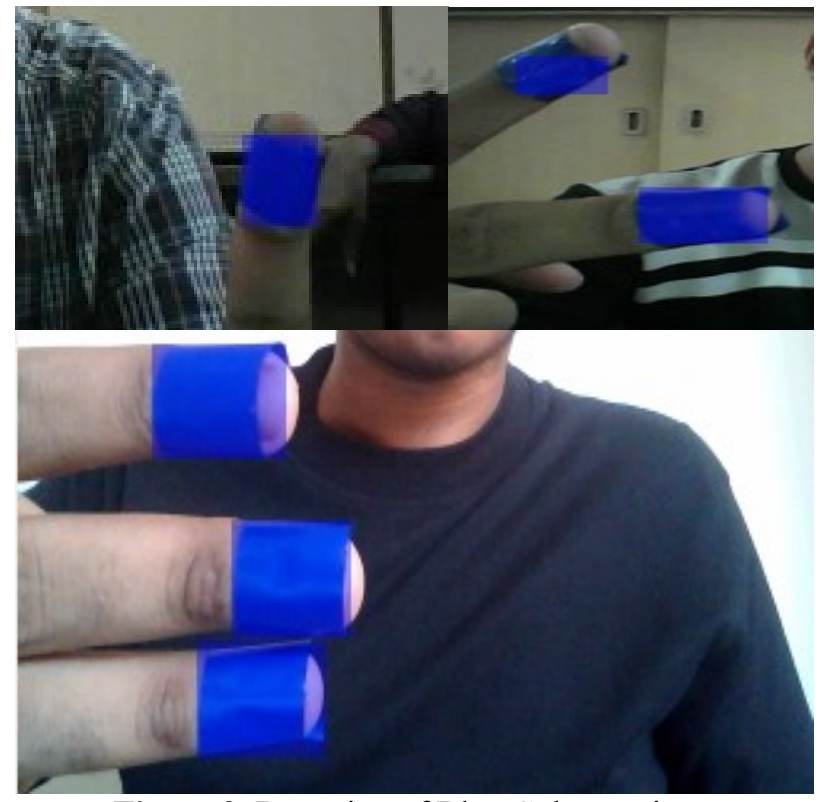

Figure 3. Detection of Blue Colour pointer

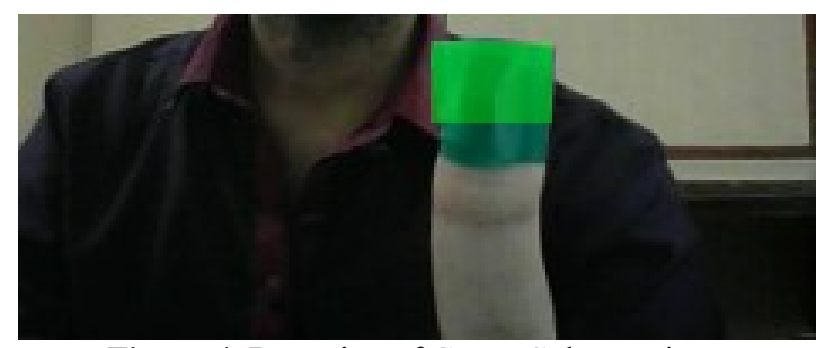

Figure 4. Detection of Green Colour pointer

\subsection{Performing Mouse Actions}

After detection of colour pointer the colour of the pointer is determined to perform the associated mouse actions. The cursor movement is controlled using red colour pointer, the clicking action is performed using blue colour pointer and the scrolling action is performed using green pointer.

When red colour pointer is detected firstly the area of detected contour is calculated. With the help of the area calculated the centroid of the detected colour is calculated. The movement of the cursor is controlled using this centroid. Then the co-ordinate of the centroid is found. To control the mouse cursor movement these co-ordinate need to be passed to the mouse driver. These co-ordinates are stored in a variable. But MATLAB cannot directly interact with the mouse driver, so JAVA is used to interact with the mouse drivers. The JAVA creates a java object which is linked with the mouse driver of the system. This object sends the co-ordinate stored in the variable. The cursor moves in accordance with the co-ordinates sent the object moves continuously, a new centroid is determined for each frame. So the cursor obtains new co-ordinates, and so the colour pointer is tracked. So when the user moves his hands in front of the camera such that the colour pointers are in the field of vision, the mouse moves proportionally across the screen.[2][4]
To perform the clicking action we use blue pointer. We can perform left click, right click and double click depending on the number of pointer detected. To perform left click action one blue pointer is required. To perform the right click action two blue pointers are required. To perform double click action three blue pointers are required. The number of pointers is detected and then corresponding actions are taken, these commands to perform clicking action is passed using JAVA object. [2][4]

Lastly the scrolling action is performed using green colour pointer. The tracking of green colour pointer is similar to that of tracking of red colour pointer. After finding the centroid of the pointer it is stored in a variable. The scrolling action is performed depending on the position of the colour pointer. If the colour pointer is in the upper half of the screen then scroll up action is performed, and if the position of the pointer is in the lower half of the screen then scroll down action is performed.

\section{PROBLEM AND DRAWBACK}

The system is based on image capture through a webcam; it depends on illumination to a certain extent. On the other hand the presence of other coloured objects in the background might cause the system to give an irregular response. This problem can be reduced by configuring the threshold values and other parameters but still we expect no bright colour. Due to complex calculations in a very small amount of time the system might run slower on certain computers. However a standard pc or laptop might meet the required computational power for optimum performance of the system. Another important fact is that if the resolution of the camera should not be too high as it may response in decreasing the system speed. Hence to overcome this problem the resolution of the camera should be less and this change can be made in the system.

\section{RESULT}

Hence we have successfully implemented our project "Virtual Mouse". The minimum distance required between the camera and the pointer is $2.5 \mathrm{inch}$ and the maximum distance is $20 \mathrm{inch}$. The above range is given for the pointer size of $1.5 \times 1.5 \mathrm{inch}$. The range may vary with the change in size of pointer. To detect colour properly Moderate intensity of light is required.The background light intensity should not be too bright or too dull as it will affect the detection of pointers.

\section{CONCLUSION}

Human Computer Interaction (HCI) is more convenient to interact with devices through computer vision. Numbers of applications for the hand gestures are being developed by researchers in recent years and they always try to minimize the use of peripheral devices that interacts with computer. Hence physically disabled people will get the most benefit out of it. So we expect that the researchers will get an extra ordinary result as an outcome of their effort. 


\section{REFERENCES}

[1] A. Erdem, E. Yardimci, Y. Atalay, V. Cetin, A. E. "Computer vision based mouse", Acoustics, Speech, and Signal Processing, Proceedings. (ICASS). IEEE International Conference, 2002

[2] Abhik Banerjee, Abhirup Ghosh, Koustuvmoni Bharadwaj, Hemanta Saikia "Mouse Control using a Web Camera based on Colour Detection"

[3] K N. Shah, K R. Rathod and S. J. Agravat, "A survey on Human Computer Interaction Mechanism Using Finger Tracking" International Journal of Computer Trends and Technology, 7(3), 2014, 174-177

[4] Hojoon Park, "A Method for Controlling the Mouse Movement using a Real Time Camera", Brown University, Providence, RI, USA, Department of Computer science, 2008

[5] Chu-Feng Lien, "Portable Vision-Based HCI - A Realtime Hand Mouse System on Handheld Devices", National Taiwan University, Computer Science and Information Engineering Department

[6] Kamran Niyazi, Vikram Kumar, Swapnil Mahe, Swapnil Vyawahare, "Mouse Simulation Using Two Coloured Tapes", Department of Computer Science, University of Pune, India, International Journal of Information Sciences and Techniques (IJIST) Vol.2, No.2, March 2012

[7] Shahzad Malik, "Real-time Hand Tracking and Finger Tracking for Interaction", CSC2503F Project Report, December 18, 2003

[8] Rafael C. Gonzalez and Richard E. Woods, Digital Image Processing, 2nd edition, Prentice Hall, Upper Saddle River, New Jersey, 07458

[9] The MATLAB website. [Online]. Available http://in.mathworks.com/discovery/imagethresholding.html

[10] The MATLAB website. [Online]. Available http://in.mathworks.com/matlabcentral/newsreader/vie w thread/140256

[11] The MATLAB website. [Online]. Available http://in.mathworks.com/matlabcentral/newsreader/vie w thread $/ 282662$

[12]The MATLAB website. [Online]. Available http://www.mathworks.com/matlabcentral/fileexchange /28757-tracking-red-color-objects-using-matlab 